\title{
Chagas Disease: in vivo Acute Infection Induces Morphological Changes in Monocytes/Macrophages But Not Nitric Oxide Production
}

\author{
Daniela L. Fabrino*, Leonor L. Leon**, Gleydes G. Parreira***, Patrícia E. Almeida** and \\ Rossana C.N. Melo* ${ }^{1}$. \\ *Laboratory of Cellular Biology, Department of Biology, Federal University of Juiz de Fora, \\ UFJF, Juiz de Fora, Minas Gerais, Brazil. \\ ${ }^{* *}$ Laboratory of Biochemistry of Tripanosomatides, Department of Immunology, Oswaldo \\ Cruz Institute, FIOCRUZ, Rio de Janeiro, RJ, Brazil. \\ **** Department of Morphology, Federal University of Minas Gerais, UFMG, Belo Horizonte, \\ Minas Gerais, Brazil.
}

Chagas disease, caused by intracellular protozoan Trypanosoma cruzi, is one of the most important public health problems in Latin America [1]. The acute infection triggers a plentiful production of monocytes from bone marrow precursor cells so as to control T. cruzi replication in the target tissues such as the myocardium [2]. Clear monocyte/macrophage activation occurs earlier in the acute $T$. cruzi infection in parallel to a high number of these cells respectively in the blood and heart $[2,3]$.

In the present work, the nitric oxide (NO) production by peripheral blood monocytes (PBM) and peritoneal macrophages was evaluated, in vivo, in rats during the acute phase of disease. The ultrastructure of the PBM and peritoneal and heart macrophages was also analyzed. Animals were inoculated with $T$. cruzi $\left(3 \times 10^{5}\right.$ tripomastigotes, i.p., Y strain) and sacrificed at days 6,12 or 20 post-infection (4-6 rats/group).

Acute T. cruzi infection did not induce NO synthesis by monocytes and macrophages despite of being found a significant increase in the number of these cells. PBM from infected animals showed ultrastructural signs of activation, being more voluminous with more euchromatic nucleus and a striking increase in cytoplasmic organelles and surface rufflings compared to controls (FIG. 1). Macrophages from infected animals showed clear morphology of activated cells. Many pseudopodia, arrays of rough endoplasmic reticulum, lipid bodies, mitochondrion profiles and vesicles were present (Fig.2). Macrophages were often seen ingesting T. cruzi forms and showed many phagolysosomes with varying sizes and electron-densities containing amorphous materials, cell debris and amastigotes. The monocyte and macrophage sizes (diameter measurements taken from electron micrographs) in infected rats were significantly higher than those of the control group cells, especially at day 12 of infection (Table 1).

Our data showing a high number of morphologically activated monocytes/macrophages highlights the importance of innate immunity during the acute Chagas disease. A novel finding is that NO production does not seem essential to control the whole period of the in vivo acute phase of $T$. cruzi infection in rats.

\section{REFERENCES}

[1] R.C.N. Melo and Machado, C.R.S. Exp. Parasitol. 97 (2001)15.

[2] C. Ropert et al., Mic.Infec. 4 (2002) 1015.

[3] R.C.N. Melo et al., Tissue and Cell. 35 (2003) 59.

[4] This research was Supported by CNPq, FIOCRUZ and FAPEMIG 
Table 1. Diameter measurements $(\mu \mathrm{m})$ from peripheral blood monocytes and peritoneal and heart macrophages in rats with 12 days of infection and controls. Values expressed as mean \pm standard deviation. The range is indicated in brackets.Monocyte/macrophage system morphometry at different days of T.cruzi infection in rats.

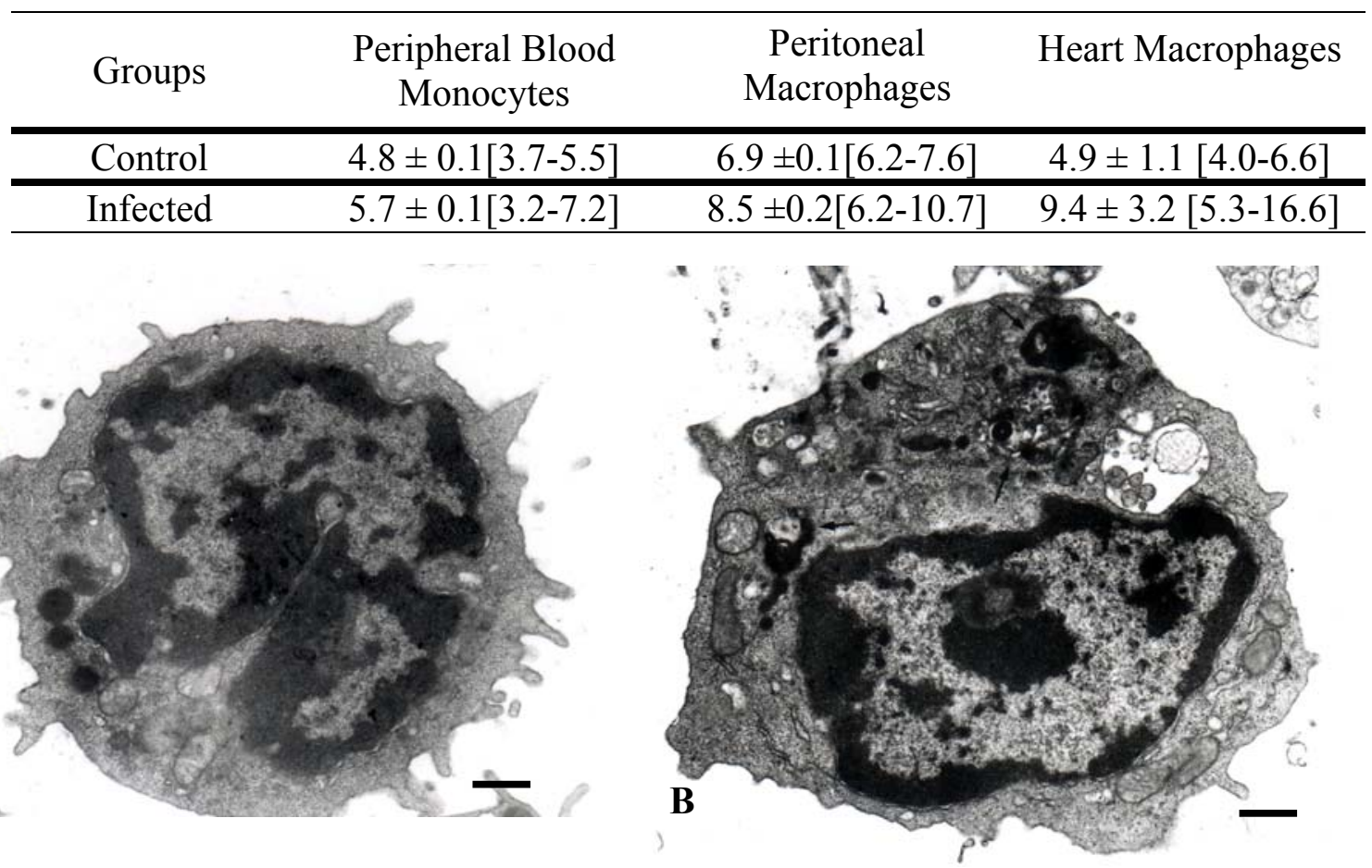

Figure 1. Electron micrographs of peripheral blood monocytes in normal (A) or infected (B) rats at day 12 day of infection with T.cruzi. Digestive vacuoles are indicated (arrows).Scale bar $=0.5 \mu \mathrm{m}$

A

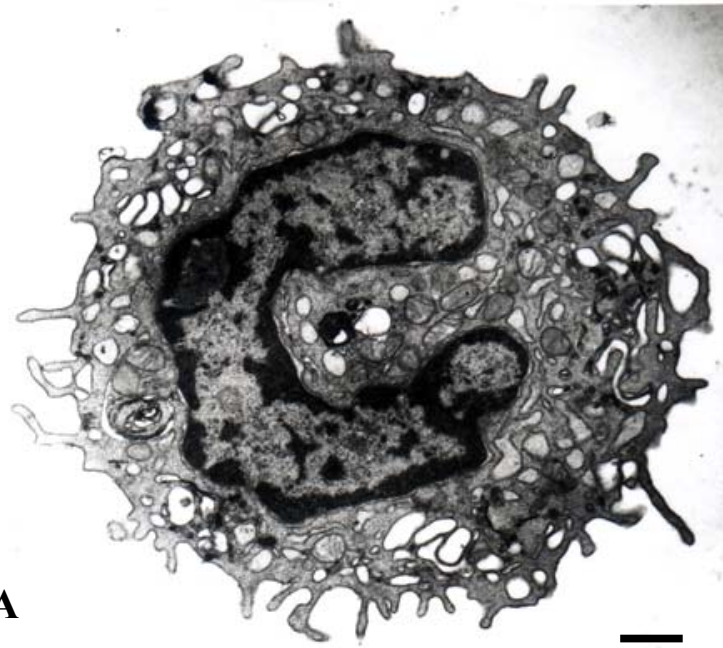

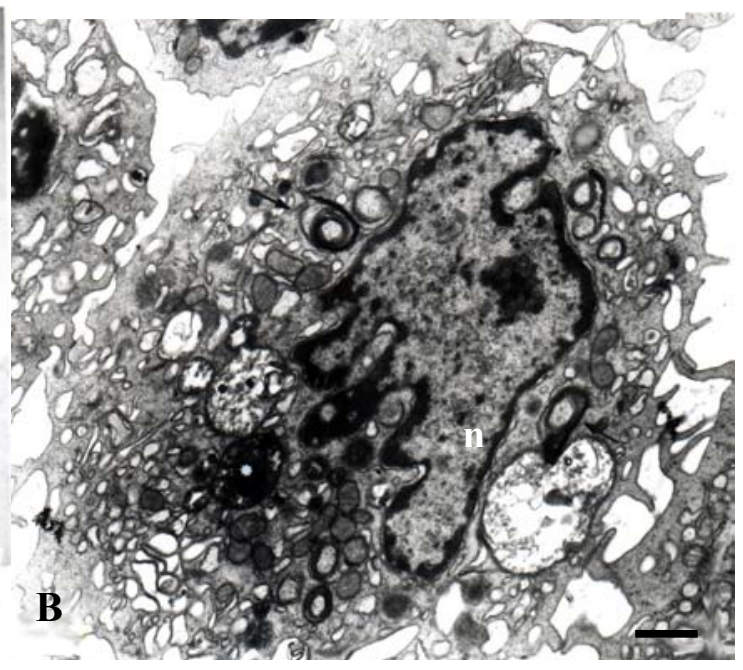

Figure 2. Electron micrographs of control (A) and infected (B) peritoneal macrophages from a rat at day 12 of infection with T.cruzi. The cells show nucleus (n) irregular in outline, voluminous cytoplasm rich in organelles and degenerating parasite amastigote forms (arrows). Scale bar $=0.5 \mu \mathrm{m}$ 